\title{
Improved spatial interpolation of rainfall using Genetic Programming
}

\author{
$\underline{\text { S. K. Adhikary }}^{\text {a }}$, A. G. Yilmaz ${ }^{\text {a, b }}$ and N. Muttil ${ }^{\text {a, b }}$ \\ ${ }^{a}$ College of Engineering and Science, Victoria University, PO Box 14428, Melbourne, VIC 8001, Australia \\ ${ }^{b}$ Institute for Sustainability and Innovation, Victoria University, PO Box 14428, Melbourne, VIC 8001, \\ Australia \\ Email: sajal.adhikary@live.vu.edu.au
}

\begin{abstract}
Rainfall data provide an important input for various water resources management tasks. Hydrologists are often required to estimate areal average rainfall over the catchment and/or point rainfall values at unsampled locations from observed sample measurements at neighboring locations. Accurate spatial distribution of rainfall can be achieved through a dense network of rain gauges. However, the rain gauge network is usually sparse in most cases and thus sufficient point rainfall measurements are not available, which are often unable to characterize the spatial distribution of highly variable rainfall. Spatial interpolation method plays an important role in such cases to estimate rainfall at unrecorded locations (i.e., missing data) using the observed rainfall available at surrounding locations.
\end{abstract}

Conventionally, variance-dependent stochastic spatial interpolation methods such as kriging are the most commonly used methods for estimating the point rainfall values at any desired locations based on the available recorded values at neighboring rain gauges. However, traditional kriging has a major weakness because it requires a priori definition of mathematical function for the variogram model that represents spatial correlations among data points and thus significantly impacts the performance of the methods. The robustness of kriging methods heavily depends on how the variogram model is constructed. Moreover, selection of appropriate variogram model, finding the optimal variogram parameters (i.e., nugget, range, sill) and the computational burden involved are some of the difficulties involved with the traditional kriging.

More recently, data-driven models using evolutionary and biological principles including genetic algorithms, artificial neural networks have been used with kriging for spatial interpolation of rainfall. Genetic programming (GP) is another evolutionary data-driven modelling technique to approximate function. The key advantage of GP is that it does not assume any a priori functional form of the solution and GP inferred models offer some possible interpretations to the underlying process. The aim of this study is to investigate the suitability of GP for variogram modelling to derive the variogram model and use of the GP-derived variogram model in combination with traditional ordinary kriging for spatial interpolation of rainfall. This new variant of kriging is referred to as genetic programming-based ordinary kriging (GPOK) in which the GP-derived variogram model replaced the standard parametric variogram models (i.e., exponential, gaussian, spherical) in the traditional ordinary kriging.

Developed genetic programming-based ordinary kriging (GPOK) method was then applied to estimate the unknown rainfall values at a rain gauge station through spatial interpolation using the historical rainfall data from 19 rain gauge stations in the Middle Yarra River catchment of Victoria, Australia. The results indicated that the GPOK method outperformed the traditional ordinary kriging method for spatial interpolation of rainfall and yielded better rainfall estimates. The results also showed that the function approximation capability of GP produces the best fitted GP-derived variogram model compared to the standard models. Moreover, variogram model fitting by GP was very quick since GP did not require identifying the variogram parameters in advance unlike the standard variogram models in the traditional kriging method. Thus, use of GP, as a universal function approximator, for variogram modelling eliminates the time consuming and tedious job of trial and error for determining the optimal variogram parameters as necessary with the standard variogram models. This results in the significant reduction in the computation complexity by the GPOK method. Therefore, the GP-derived variogram model seems to be a potential alternative to variogram models used in the past and the proposed GPOK method is recommended as a viable option for improved spatial interpolation.

Keywords: Genetic programming, ordinary kriging, variogram model, rainfall data, spatial interpolation 


\section{INTRODUCTION}

Rainfall data provide an essential input for most hydrologic analyses and designs for effective management of water resources systems. Accurate spatial distribution of rainfall can be achieved through a dense network of rain gauges. However, the rain gauge network is usually sparse in most cases and thus point rainfall measurements are not sufficient to characterize the spatial distribution of highly variable rainfall (Adhikary et al., 2015). Spatial interpolation method plays a vital role in such cases to estimate rainfall at unrecorded locations (i.e., missing rainfall data) using the observed rainfall available at surrounding locations.

Deterministic, stochastic and data-driven interpolation methods have been employed in the past to estimate the missing rainfall data. Deterministic methods such as inverse-distance weighting have been extensively used for estimating missing rainfall (ASCE, 1996). Although they have been improved over time, their limitations continue to exist (Teegavarapu, 2007). Stochastic interpolation method such as the kriging is another commonly used method. In particular, ordinary kriging $(\mathrm{OK})$ remains one of the most preferred methods, which has been widely used to interpolate point rainfall measurements for missing rainfall estimation (Webster and Oliver, 2007). However, the main drawback of kriging is that it is highly dependent on the variogram model that defines the spatial variability of the data. Moreover, selection of appropriate variogram model, finding the optimal variogram parameters (i.e., nugget, range, sill) and the computational burden involved are some of the difficulties associated with the traditional kriging.

More recently, data-driven models using evolutionary and biological principles, namely genetic algorithms (GA), artificial neural networks (ANN), adaptive neuro-fuzzy inference system, and support vector regression, have been used with kriging for spatial interpolation of rainfall and missing rainfall estimation (Chang et al., 2005; Teegavarapu, 2007; Teegavarapu et al., 2009; Kisi and Sanikhani, 2015). However, ANN and GA usually do not provide analytical equations, and it is generally not easy to interpret the network weights obtained from the obtained models by ANN (Muttil and Lee, 2005).

Genetic programming (GP) (Koza, 1992) is another evolutionary data-driven modelling technique, which can be used for function approximation. GP inferred models have the advantages of generating simple expressions and thus offering some possible interpretations to the underlying process (Muttil and Lee, 2005). The key advantage of GP is that it does not assume any a priori functional form of the solution. Several applications of GP in water resources research have been reported, which include rainfall-runoff simulation (Khu et al., 2001; Liong et al., 2002; Yilmaz and Muttil, 2014), prediction of algal blooms (Muttil and Lee, 2005; Sivapragasam et al., 2010), friction factor simulation (Roushangar et al., 2014), sediment transport modelling (Babovic and Abbott, 1997), vegetation effect on flow in wetlands (Harris et al., 2003). To the best knowledge of the authors, GP has not been applied for variogram modelling in the past and hence the aim of this study is to apply GP for variogram modelling to be used with kriging.

In this study, a new variant of kriging method with the combination of GP-derived variogram model and OK was proposed and evaluated for estimating missing rainfall data at a gauging station located in the Middle Yarra River catchment, Australia. The proposed form of kriging is referred to as genetic programming-based ordinary kriging (GPOK) in which the standard parametric variogram models (i.e., exponential, gaussian, spherical) used with the traditional OK were replaced with the GP-derived variogram model.

The paper first presents details of the study area and data used for the analysis in Section 2, which is followed by a brief description of traditional $\mathrm{OK}$ and proposed GPOK methods with their application for missing rainfall estimation in Section 3 and Section 4. The results and discussion are then presented in Section 5 and finally, the conclusions are drawn in Section 6.

\section{STUDY AREA AND DATA USED}

The Yarra River catchment in Victoria, Australia covering an area of $4044 \mathrm{~km}^{2}$ has three distinctive subcatchments: Upper Yarra, Middle Yarra, and Lower Yarra segments (Adhikary et al, 2015). The middle segment of the catchment with an area of $1511 \mathrm{~km}^{2}$ was selected as the case study area, which is shown in Figure 1. Accurate rainfall estimation is vital for the precise computation of future streamflows for optimal reservoir operation in the Upper and Middle segments and effective flood controls in the lower urbanized segment.

Historical daily rainfall data for 1980-2012 (12054 days) period from 19 rain gauges operated by the Bureau of Meteorology (BoM), Australia in the Middle Yarra River catchment (Figure 1) were used for the analysis. The average annual rainfall based on the collected data varied between $508.1 \mathrm{~mm}$ to $1913.9 \mathrm{~mm}$ with the highest rainfall in the southern, eastern and north-eastern part and the lowest rainfall in north-western part of the study area. The wettest month is September (rainfall equals to $112.5 \mathrm{~mm}$ ) with the highest rainfall 
variation and February is the driest month (rainfall equals to $56.4 \mathrm{~mm}$ ) with the second highest rainfall variation. The elevation varies from $25 \mathrm{~m}$ (lowest-mainly in the central, north-western and western part) to $1243 \mathrm{~m}$ (highest-mainly in the northern, north-eastern and eastern part) with a mean elevation of $621 \mathrm{~m}$ above mean sea level.

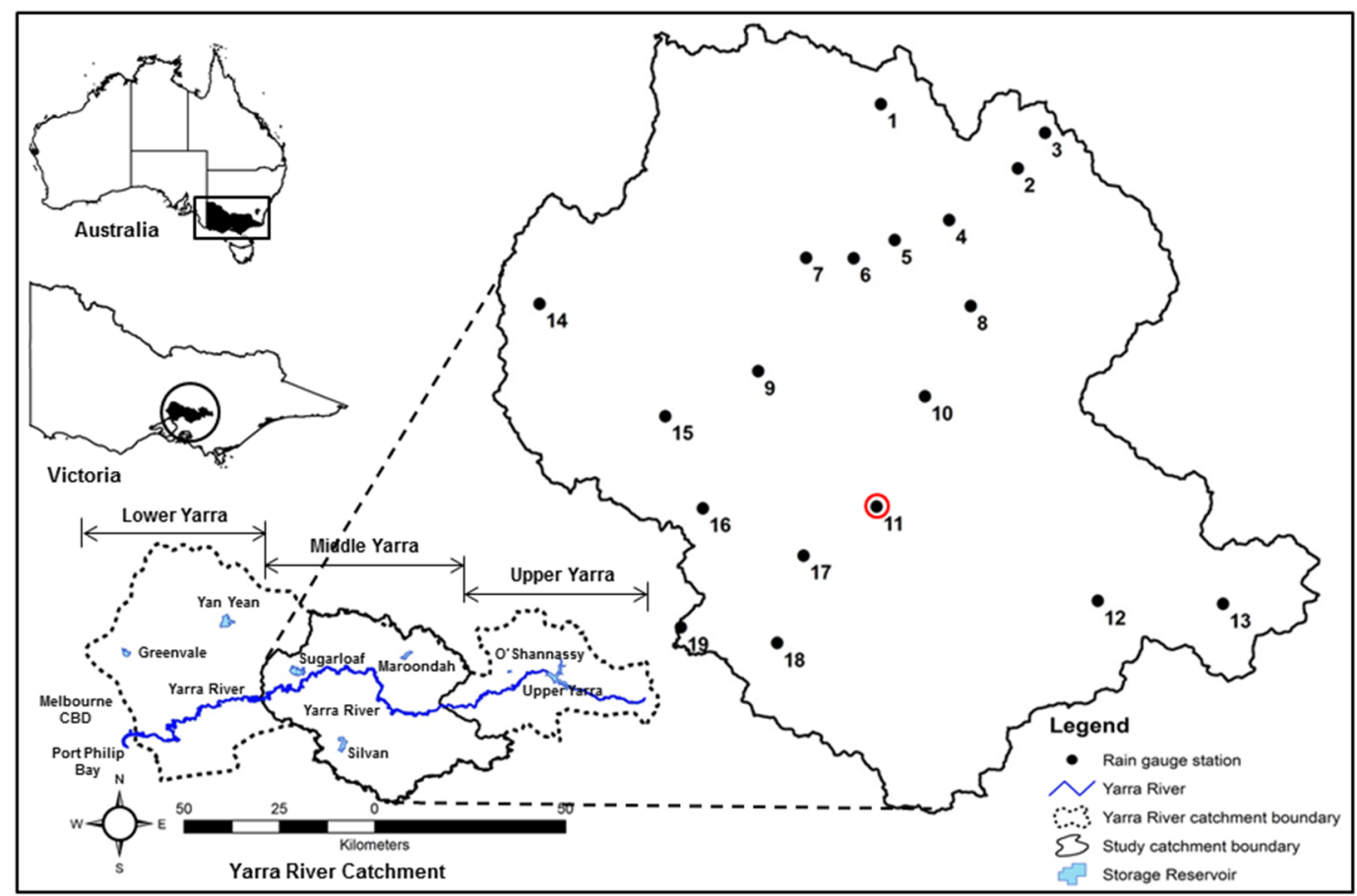

Figure 1. The study area (Middle Yarra River catchment) showing the rain gauge stations (station 11 (BoM ID: 86367, Seville) is the base station at which the missing rainfall data are to estimated)

\section{ORDINARY KRIGING}

Kriging refers to a surface interpolation technique based on spatially dependent variance, which estimates variable values at desired locations in space using the known sampling values (Webster and Oliver, 2007). In this study, OK was used for the spatial interpolation of point rainfall data for missing rainfall estimation, which is known as the best linear unbiased estimator of a variable at unsampled location with an unknown constant mean (Teegavarapu, 2007).

The general expression of $\mathrm{OK}$ to estimate missing value of a variable $Z$ (rainfall in this study) at desired location $x_{0}, Z_{O K}^{m}\left(x_{0}\right)$ in space is given by:

$$
Z_{O K}^{m}\left(x_{0}\right)=\sum_{i=1}^{n} w_{i}^{O K} Z\left(x_{i}\right) \quad \text { where } \sum_{i=1}^{n} w_{i}^{O K}=1
$$

Where $w_{i}^{O K}$ is the kriging weights associated with the observation at location $x_{i}$ with respect to $x_{0}$, which is usually determined based on the variogram model, and $n$ indicates the number of observed data points.

The kriging technique highly depends on the variogram model that defines the spatial structure of the data. The variogram model gives variogram values for any distance and allows a unique and stable solution for the kriging weights. Traditional OK method generally uses standard variogram models $\gamma_{S t d}(h)$. In this study, three most commonly used variogram models in hydrology, namely, exponential, gaussian, spherical models were considered. Initially, an experimental variogram was derived based on the observed rainfall and then variogram model was fitted to the experimental variogram. Fitting of variogram model was performed by GS+ software (Robertson, 2008). The model fitted with the lowest root-mean-square error (RMSE) and mean absolute error (MAE), and the highest coefficient of correlation (CC) was chosen as the best variogram model. Finally, the adequacy of the adopted standard variogram model was tested by a leave-one-out crossvalidation procedure. In this study, mean standardized error (MS), root-mean-square error (RMSE), mean kriging standard error (MKSE), and root-mean-square standardized error (RMSS) were used as crossvalidation statistics. MS, RMSE and MKSE close to 0, and RMSS close to 1 indicate that the variogram model is satisfactory to yield accurate interpolation results by kriging (Adhikary et al., 2015). 


\section{GENETIC PROGRAMMING-BASED ORDINARY KRIGING}

In this study, the GP-derived variogram model was combined with the traditional OK to form the proposed GPOK method. The conceptual difference between the traditional OK and the proposed GPOK methods is shown in Figure 2. As can be seen in the figure, the variogram model derived by GP replaces the standard variogram models in the traditional OK to develop the proposed variant of kriging, which was referred to as the GPOK method. The main advantage of the proposed GPOK method is that the GP-derived variogram model does not require identifying the variogram parameters in advance unlike the standard variogram models and thus the GPOK method is free from tedious trial and error task of finding the parameters.

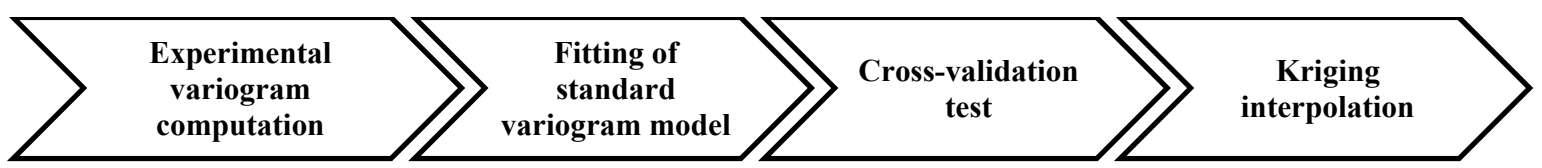

(a) Traditional ordinary kriging (OK) method

$\sum \begin{gathered}\text { Experimental } \\ \text { variogram } \\ \text { computation }\end{gathered}\left|\begin{array}{c}\text { Variogram model } \\ \text { fitting by genetic } \\ \text { programming }\end{array}\right| \begin{gathered}\text { Cross-validation } \\ \text { and positive } \\ \text { definiteness test }\end{gathered}\left|>\begin{array}{c}\text { Kriging } \\ \text { interpolation }\end{array}\right\rangle$

(b) Genetic programming-based kriging (GPOK) method

Figure 2. Conceptual difference between the traditional ordinary kriging and GPOK methods

The general expression of GPOK to estimate missing value of variable $Z$ (rainfall in this study) at desired location $x_{0}, Z_{G P O K}^{m}\left(x_{0}\right)$ in space is given by:

$Z_{G P O K}^{m}\left(x_{0}\right)=\sum_{i=1}^{n} w_{i}^{G P O K} Z\left(x_{i}\right) \quad$ where $\sum_{i=1}^{n} w_{i}^{G P O K}=1$

Where, $w_{i}^{G P O K}$ is the kriging weights associated with the observation at location $x_{i}$ with respect to $x_{0}$, which is determined based on the GP-derived variogram model $\gamma_{G P}(h) . \gamma_{G P}(h)$ is the variogram value that comes from the GP-derived variogram model.

In this study, GP was used as a universal functional approximator to fit the experimental variogram in order to obtain the GP-derived variogram model. GP is a relatively new automatic programming technique for evolving computer programs to solve problems (Koza, 1992). In engineering applications, GP is frequently used as a universal function approximator to approximate and interpret the underlying structure of either a natural or experimental process (Muttil and Lee, 2005). What makes GP unique compared to the traditional data-driven methods is that it provides the underlying structure of the process in the form of a mathematical function (or equation), which can be scientifically interpreted to derive knowledge about the process to be modelled. Moreover, it does not assume any a priori functional form of the solution.

The basic search strategy behind GP (Koza, 1992) is a GA. It differs from the traditional GA in that it usually operates on parse trees instead of bit strings to approximate the equation that best describes how the output are related to the input variables. A parse tree is formed by a terminal set (the variables involved in the problem) and a function set (the basic operators used to form the function). As a GA, GP proceeds by initially generating a population of random parse trees, calculate their fitness - a measure of how well they solve the given problem and subsequently selects the better parse trees for reproduction and recombination to form a new population. This process of selection and reproduction iterates until some stopping criteria is satisfied. The recombination takes place by crossover and mutation. For a comprehensive description of GP from water resources perspective, the readers are referred to Babovic and Keijzer (2000), Khu et al. (2001), Muttil and Lee (2005).

Mathematically, the GP-derived variogram model $\gamma_{G P}(h)$ in this study is given by:

$$
\gamma_{G P}(h)=f(\text { Average lag distance between stations, } h \text { ) }
$$

In this study, GPKernel, developed by DHI Water and Environment (Babovic and Keijzer, 2000) was used for implementing GP to obtain the GP-derived variogram model. The GPKernel is a command line based tool for finding the underlying functions (in mathematical form) on data. The model with the lowest RMSE and MAE values and the highest CC value was chosen as the best GP-derived variogram model, which was finally checked by the cross-validation and positive definiteness test before using in kriging. The positive definiteness condition for a variogram model demonstrates that the variance obtained based on the variogram 
model remains always positive (Webster and Oliver, 2007). Teegavarapu (2007) mentioned that developing and selecting a new variogram model (for example, the GP-derived variogram model in this study) other than the standard ones may not result in a unique and stable solution for the kriging weights, if the positive definiteness condition is not satisfied. He also indicated that only the standard variogram models (i.e., exponential, gaussian, spherical) usually satisfy the positive definiteness condition. Therefore, the GPderived model should satisfy the cross-validation and positive definiteness tests simultaneously before it can be used for spatial interpolation of rainfall by the GPOK method.

\section{RESULTS AND DISCUSSION}

\subsection{Modelling Experimental Variogram by Standard Variogram Models}

For variogram modelling, first an experimental variogram was computed based on the mean daily rainfall data. Initially, the directional variograms in the $\mathrm{X}$ and $\mathrm{Y}$ directions were computed. However, they were found to be noisy due to lack of sufficient data points in these directions. Therefore, an isotropic experimental variogram was computed ignoring the effect of anisotropy on variogram parameters. Isotropy refers to a property that indicates the spatial autocorrelation between two locations changes only with the distance (Webster and Oliver, 2007).

The experimental variogram was then modelled by three standard variogram functions (i.e., exponential, gaussian, spherical models in this study). The variogram parameters (i.e., nugget, range, and sill) were iteratively changed using GS+ software to obtain the best fitted model. The parameters that resulted in the best fitting performance based on RMSE, MAE, and CC values were identified as the optimal variogram parameters and the corresponding models were selected as the best fitted variogram model. The functional forms obtained for the standard variogram models are given in Table 1. These standard variogram models provide variogram values $\gamma_{s t d}(h)$ to determine the kriging weights in Equation (1) in traditional OK. Table 2 shows the fitting performance and cross-validation results of the fitted models, which indicate that the gaussian model is the best fitted standard variogram model considering all three measures. The results also indicate that these models satisfy all the cross-validation criteria and are thus appropriate for use in kriging.

Table 1. Functional forms obtained for different variogram models

\begin{tabular}{ll}
\hline Variogram model & Functional forms \\
Standard variogram model & \\
Exponential & $\gamma(h)=0.001+0.460\left[1-\exp \left(-\frac{3 h}{36.96}\right)\right]$ \\
Gaussian & $\gamma(h)=0.035+0.344\left[1-\exp \left(-\frac{3 h^{2}}{(16.14)^{2}}\right)\right]$ \\
Spherical & $\gamma(h)= \begin{cases}0.001+0.381\left[\frac{1.5 h}{20.38}-\frac{0.5 h^{3}}{(20.38)^{3}}\right] & \text { for } h \leq 20.38 \\
0.382 & \text { for } h>20.38\end{cases}$ \\
Variogram model by $\boldsymbol{G P}$ & $\begin{array}{l}0.023+0.344\left[1-\exp \left(-\frac{3 h^{2}}{(16.14)^{2}}\right)\right] \\
\text { GP-derived }\end{array} \quad$ for $h<4.393$ \\
\hline
\end{tabular}

Note: $h$ is the distance in $\mathrm{km}$

\subsection{Modelling Experimental Variogram by Genetic Programming}

For modelling experimental variogram using GP, simple function sets $\left(+,-, *, /, x^{2}\right.$, exp) exists in the standard variogram functions (Table 1) were adopted for the GP runs in order to generate simple GP-derived variogram models. It was intended that the evolved GP models based on these function sets could exhibit a similar structure of the standard variogram models. Minimization of the RMSE was used as the objective function for the evolution of best fitted model by GP. In this study, the optimal functional form of the GPderived variogram model was obtained using a population size of 500, crossover rate of 0.95 , mutation rate of 0.05 and the number of generations as 500 .

The functional form of the GP-derived variogram model is given in Table 1, which provides variogram values $\gamma_{G P}(h)$ for computing the kriging weights in Equation (2) in GPOK. It can be also seen from Table 1 that the GP-derived variogram model consists of a simple structure similar to that of the standard variogram 
models. This indicates the advantage of GP over other traditional data-driven methods such as ANN for variogram modelling because of its ability to build models with a simple structure. Moreover, fitting of the experimental variogram by GP was very quick since GP did not require a pre-defined mathematical form or architecture unlike ANN to generate the variogram model. Furthermore, the GP-derived variogram model did not require identifying the variogram parameters in advance unlike standard variogram models. As a result, variogram modelling by GP was free from the trial and error process of estimating the variogram parameters. Hence, the GP-derived variogram model seems to be an alternative to the standard variogram models.

Table 2. Performance measures and cross-validation statistics for fitted variogram models used in kriging

\begin{tabular}{|c|c|c|c|c|c|c|c|}
\hline \multirow{2}{*}{ Variogram model } & \multicolumn{3}{|c|}{ Goodness-of-fit measures } & \multicolumn{4}{|c|}{ Cross-validation statistics } \\
\hline & RMSE & MAE & $\mathrm{CC}$ & MS & RMSE & MKSE & RMSS \\
\hline Exponential & 0.0268 & 0.0211 & 0.972 & -0.1006 & 0.4691 & 0.4500 & 1.0424 \\
\hline Gaussian & 0.0227 & 0.0187 & 0.978 & -0.1100 & 0.5403 & 0.5500 & 0.9824 \\
\hline Spherical & 0.0247 & 0.0211 & 0.974 & -0.1215 & 0.3922 & 0.3960 & 0.9905 \\
\hline GP-derived & 0.0204 & 0.0155 & 0.982 & -0.0155 & 0.3582 & 0.3840 & 0.9328 \\
\hline
\end{tabular}

As can be seen from Table 3, the GP-derived variogram model satisfied all the cross-validation criteria and also resulted in the best fitting performance over the standard variogram models. In order to test the positive definiteness condition, the kriging variance based on the GP-derived variogram model was calculated for all 19 rain gauge locations, which was found positive. This confirms that the GP-derived model and its corresponding covariance function satisfy the positive definiteness condition. Thus, the GP-derived model is capable of providing a unique and stable solution for the kriging weights in GPOK method.

\subsection{Spatial Interpolation of Rainfall by Traditional OK, and GPOK methods}

In this study, the traditional OK and proposed GPOK methods were applied to estimate missing rainfall data at a base station (i.e., Seville, station no. 11 in Figure 1). The base station refers to a station with missing data where the estimation is desired through spatial interpolation of the data available from the surrounding stations. In order to test the robustness of the proposed GPOK method, it was assumed that the data were missing at the base station (although the data were available). The traditional OK was implemented through GS + software and the proposed GPOK was implemented through a customized spreadsheet application developed in the Microsoft Excel platform. The performance of the traditional OK and proposed GPOK methods was compared using the commonly used and widely recognized error measures, namely, RMSE and MAE based on the observed and estimated rainfall data at the base station.

Two-third (i.e., 8000 days) of the total historical data (i.e., 12054 days) were used for estimating the kriging weights and the missing rainfall was estimated for the rest one-third (i.e., 4054 days) data and the performance of both methods was compared. In case of traditional OK method, the three standard variogram models as provided in Table 1 were used to determine the kriging weights and thus estimate the missing rainfall data at the base station using Equation (1) through spatial interpolation. In case of GPOK method, the GP-derived variogram model as presented in Table 1 was used to determine the kriging weights and thus estimate the missing rainfall data at the base station using Equation (2) through spatial interpolation. The kriging variance was also estimated by the GPOK method at the base station (i.e., Seville, station no. 11), which was found as $0.175 \mathrm{~mm}^{2}$. The variance is positive and hence the pre-requisite positive definiteness condition was satisfied by the GP-derived variogram model and there the GPOK method.

Table 3. Performance of traditional ordinary kriging and GPOK methods for spatial interpolation of rainfall

\begin{tabular}{lcccc}
\hline Method & RMSE & $\begin{array}{c}\text { Improvement of } \\
\text { RMSE by GPOK (\%) }\end{array}$ & MAE & $\begin{array}{c}\text { Improvement of } \\
\text { MAE by GPOK (\%) }\end{array}$ \\
\hline $\mathrm{OK}^{\mathrm{E}}$ & 1.722 & 11.6 & 0.583 & 23.5 \\
$\mathrm{OK}^{\mathrm{G}}$ & 1.653 & 7.9 & 0.547 & 18.5 \\
$\mathrm{OK}^{\mathrm{S}}$ & 1.734 & 12.2 & 0.603 & 26.1 \\
$\mathrm{GPOK}$ & 1.522 & - & 0.446 & - \\
\hline
\end{tabular}

Note: $\mathrm{OK}^{\mathrm{E}}$ - $\mathrm{OK}$ with the exponential variogram model; $\mathrm{OK}^{\mathrm{G}}-\mathrm{OK}$ with the gaussian variogram model

$\mathrm{OK}^{\mathrm{S}}$ - OK with the spherical variogram model; GPOK - OK with the GP-derived variogram model

Results obtained for the performance of the aforementioned traditional OK and proposed GPOK methods are presented in Table 3. As can be seen from the table that OK with the gaussian variogram model yielded the best performance when compared to $\mathrm{OK}$ with remaining two standard variogram models (i.e., exponential, spherical). It is also seen that the performance of the proposed GPOK method outperformed the traditional OK with all the three standard variogram models for estimating the missing rainfall values at the base station. 
When comparing the performance of the GPOK method with the best traditional kriging (OK with the gaussian variogram model) method, a reduction in error of $7.9 \%$ for RMSE and $18.5 \%$ for MAE was obtained with the GPOK method. Therefore, it can be concluded that use of the GPOK over traditional OK method results in a significant reduction of error for missing rainfall estimation, and thereby provides more accurate estimates of rainfall at the base station.

\section{CONCLUSIONS}

In this study, a new spatial surface interpolation method was proposed, which is referred to as the genetic programming-based ordinary kriging (GPOK) method. In the proposed GPOK method, GP was used as a universal function approximator to obtain the GP-derived variogram model that was used as an alternative to the standard variogram models in traditional OK. This study finally evaluates the efficacy and robustness of the newly proposed GPOK method over traditional OK method through a case study aimed at estimating missing rainfall data with the objective of minimizing the estimation error. The proposed GPOK method provided the best performance of the spatial surface interpolation when compared to the traditional OK method. Therefore, this study concludes that the fusion of geostatistical (ordinary kriging) and data-driven (GP) techniques is effective and has a high potential for spatial surface interpolation.

\section{ACKNOWLEDGMENTS}

The authors would like to thank DHI Water and Environment for providing GPKernel and Bureau of Meteorology (BoM), Australia for providing the necessary data.

\section{REFERENCES}

Adhikary, S.K., Yilmaz, A.G., and Muttil N. (2015). Optimal design of rain gauge network in the Middle Yarra River catchment. Hydrological Processes, 29(11), 2582-2599.

ASCE. (1996). Hydrology handbook (2nd Ed.), Task Committee on Hydrology Handbook (Management Group D of ASCE), American Society of Civil Engineers (ASCE), New York, USA.

Babovic, V., and Abbott, M.B. (1997). The evolution of equations from hydraulic data, part II: applications. Journal of Hydraulic Research, 35(3), 411-430.

Babovic, V., and Keijzer, M. (2000). Genetic programming as a model induction engine. Journal of Hydroinformatics, 2, 35-60.

Chang, C.L., Lo, S.L., and Yu, S.L. (2005). Applying fuzzy theory and genetic algorithm to interpolate precipitation. Journal Hydrology, 314(1-4), 92-104.

Harris, E.L., Babovic, V., Falconer, R.A. (2003). Velocity prediction in compound channels with vegetated floodplains using genetic programming. International Journal of River Basin Management, 1(2), 117-123.

Khu, S.T., Liong, S.Y., Babovic, V., Madsen, H., and Muttil, N. (2001). Genetic programming and its application in real-time runoff forecasting. Journal of American Water Resources Association, 37(2), 439-451.

Kisi, O., and Sanikhani, H. (2015). Prediction of long-term monthly precipitation using several soft computing methods without climatic data. International Journal of Climatology, doi: 10.1002/joc.4273.

Koza, J.R. (1992). Genetic Programming: On the Programming of Computers by Means of Natural Selection, MIT Press, Cambridge, MA.

Liong, S.Y., Gautam, T.R., Khu, S.T., Babovic, V., Keijzer, M., and Muttil, N. (2002). Genetic programming: a new paradigm in rainfall runoff modelling. Journal of American Water Resources Association, 38(3), 705-718.

Muttil, N., and Lee, J.H.W. (2005). Genetic programming for analysis and real-time prediction of coastal algal blooms. Ecological Modelling, 189(3-4), 363-376.

Robertson, G.P. (2008). GS+: Geostatistics for the Environmental Sciences, Gamma Design Software, USA.

Roushangar, K., Mouaze, D., and Shiri, J. (2014). Evaluation of genetic programming-based models for simulating friction factor in alluvial channels. Journal of Hydrology, 517, 1154-1161.

Sivapragasam, C., Muttil, N., Muthukumar, S., and Arun, V.M. (2010). Prediction of algal bloom using genetic programming. Marine Pollution Bulletin, 60(10), 1849-1855.

Teegavarapu, R.S.V. (2007). Use of universal function approximation in variance-dependent surface interpolation method: an application in hydrology. Journal of Hydrology, 332(1-2), 16-29.

Teegavarapu, R.S.V., Tufail, M., and Ormsbee, L. (2009). Optimal functional forms for estimation of missing precipitation records. Journal of Hydrology, 374(1-2), 106-115.

Webster, R., and Oliver, M.A. (2007). Geostatistics for Environmental Scientists (2nd Ed.), John Wiley \& Sons, Chichester, UK.

Yilmaz, A.G., and Muttil, N. (2014). Runoff estimation by machine learning methods and application to the Euphrates basin in Turkey. Journal of Hydrologic Engineering, 19(5), 1015-1025. 